\title{
Fennoscandian strain rates from BIFROST GPS: A gravitating, thick-plate approach
}

\author{
H.-G. Scherneck ${ }^{*, a}$, M. Lidberg ${ }^{\mathrm{b}}$, R. Haas ${ }^{\mathrm{a}}$, Jan M. Johansson ${ }^{\mathrm{a}}$, G.A. Milne \\ ${ }^{a}$ Chalmers University of Technology, Dept. of Radio and Space Science, SE-412 96 Göteborg, Sweden \\ ${ }^{b}$ National Land Survey of Sweden, SE-801 82 Gävle, Sweden \\ ${ }^{c}$ University of Ottawa, Dept. of Geodsciences, Ottawa, Ont., Canada
}

\begin{abstract}
The aim of this investigation is to develop a method for the analysis of crustal strain determined by station networks that continuously collect measurements of Global Navigation Satellite Systems (GNSS). The major new ingredient is that we require a simultaneous minimum of the observation error and the elastic and potential energy of a thick elastic and gravitating slab. From this outset the method pursues a general approach not limited to the origin of the deforming process nor to assumptions of lateral homogeniety of the elastic and density structure. One present limitation, however, is the assumption of vertical uniformity in elasticity, which is relevant in the flexure mode.

The observations that we analyse come from eight years worth of daily solutions from continuous BIFROST GPS measurements in the permanent networks of the Nordic countries and their neighbours. Reducing the observed three-dimensional velocities with best-fitting predictions for the effects of glacial isostatic adjustment (GIA) we find strain rates of typically 4-6 nano/yr in the interior of the rebound area, shear strain being slightly more pronounced compared to areal strain. The largest strain rates, 12 nano/yr, accompanied by a curl feature of $4 \mathrm{nrad} / \mathrm{yr}$, are found in the Finnmarken area where, however, the GNSS network density is much lower than in the central and southern parts. The somewhat poor network geometry is found to create spurious curl at 1 to $2 \mathrm{nrad} / \mathrm{yr}$ in the Lapland-Finnmarken area.

We also find that the strain generated by flexure due to GIA is important. In th emodel, the extensional regime seen at the surface turns over into a compressive style already at moderate depth, some $50 \mathrm{~km}$.
\end{abstract}

Key words:

Space Geodesy, Recent Crustal Motions, Glacial Isostatic Adjustment

\section{Introduction}

In application to contemporary deformation observed with space geodetic techniques, the computation of strain rates has so far mostly been done on the basis of kinematic and/or stochastic

\footnotetext{
${ }^{*}$ Corresponding author

Email address: hgs@ chalmers. se (H.-G. Scherneck)
} 
concepts, and the potential importance in the vertical motion component has been mentioned only in passing (Cai and Grafarend, 2007). The majority of earlier studies has concentrated on strain accumulation at fault zones (e.g. Spakman and Nyst (2002); Nyst and Thatcher (2004)) in problems dominated by horizontal motion.

The situation in the area of glacial isostatic adjustment (GIA) for instance in Fennoscandia differs in many respects. Vertical motion is on the order of $10 \mathrm{~mm} / \mathrm{yr}$, almost an order of magnitude greater than the horizontal motion. Seismicity is low in the central areas (excepting the Norwegian shelf), typically one magnitude-four event per year (Ahjos and Uski, 1992). According to Wells and Coppersmith (1994), associated epicentral surface displacements are $0.01 \mathrm{~m}$ at most. Several hundred of such earthquakes would be needed to add up to fault-zone motion that a GNSS network could detect. Thus the field of motion does not appear to be substantially modulated by individual fault zones.

While the method of Spakman and Nyst (2002) produces grids that are rather independent of the network of observing stations, the work of other authors, including the VLBI results of Haas et al. (2002) and investigations of the ITRF station network by Nocquet et al. (2005) present their strain rates on triangulations directly dependent on the site locations, with the consequence that the area is covered with a highly irregular grid; the strain rates are treated as constants in each triangle. One thought behind the present study is to facilitate interpolation of strain rates on the basis of continuous polynomials, using elasto-mechanical constraints rather than stochastic methods.

Continuous observations from the BIFROST GNSS network in Fennoscandia are available starting in 1993. Recent comparisons of inferred rates of motion with GIA models ((Milne et al., 2004); (Lidberg et al., 2006);(Lidberg et al., 2009)) suggest that the observations can successfully be reconciled at a level of 5 of the weighted $\chi^{2}$ of fit. This leaves little signal for further investigation of systematic deficiencies of the model or systematic errors in the estimated station velocities. Nevertheless this paper will pursue an attempt to determine areas where the misfit might show patterns pertaining over scales wider than the local site scale. Rates of strain and curl will be our target quantities, since strain rates are related to stress and thus significant with respect to regional seismicity, and curl rates cannot easily be traced to GIA for the reason that the normal stress boundary conditions of ice and ocean loads on a radially symmetric body are inefficient generators of curl. Thus, curl is an indicator of either a non-GIA origin of some part of the observable deformation, or that departures from radial symmetry are significant in the real earth.

The following concept is employed. The elastic equations for a thick plate are formulated. We take into account that the plate is part of a spherical shell. The plate is stretched and flexed such that the station velocities are matched, minimising a cost function in an overdetermined system of observation equations. Free slip boundary conditions allow the plate a maximum degree of freedom. Vertical strain is neglected. The cost of deformation comprises elastic and potential energy, and the observation misfit. Strain and curl rates result as derivate products. The relation between a measure of elastic energy and the velocity field will be addressed.

In one view the thick-plate adjustment may just serve as an interpolation method for site velocities. This would correspond to a conservative attitude, being cautious as to some of the basic assumptions, in particular whether the free slip boundary condition and the homogeneous structure of the slab are realistic. The diametrical view, expecting the model's 3-D deformation to be sufficiently consistent with an elastic lithosphere, is probably over-optimistic. One virtue of the approach might be seen in its independence of a GIA assumption. We employ this notion at the stage where we analyse that part of the GNSS observations that deviates from a best-fitting 
model for GIA. Ideally, the GIA model would predict the GIA related part of the deformation, and the residual would, besides measurement noise, contain the effects of deformation of a nonGIA origin. We also derive strain and curl rates on the basis of the displacement rates from the GIA model as a test whether the procedure generates artefacts, curl in particular, due to the sparseness of the GNSS network.

\section{Model}

We define a penalty function consisting of the normalised RMS of the misfit of the 3Dmotion, elastic energy and gravitational energy. The elastic energy comprises in-plane and bending stresses and strains. The gravitational energy sums up contributions of the buoyancy of displaced layers, involving not only the thick plate itself but also and the mantle below. We have taken a small series of density jumps (at surface, crust-mantle at $200 \mathrm{~km}$, upper-lower mantle at $700 \mathrm{~km}$ ). A scaling factor must be devised that trades off the observation misfit against the energy in the elastic and buoyant features.

The buoyant property has been found important to include. Without it, the model accomplishes heavy warping in areas where measurements are not available.

Stress and strain are formulated in a spherical geometry. The base functions chosen consist of a three-set of 2D Chebyshev polynomials for the observables, the vertical and horizontal displacement rates. The polynomials have the latitude and longitude angles as independent variables. The square interval (lower left and upper right corner) $[(-1,-1),(1,1)]$ is mapped to a quadrangle containing the area of interest. Strain rate is derived from deformation rate using the appropriate formulas from differential geometry. The effects from bending of the thick plate are formulated using only the leading terms, which are those known from plane geometry.

The covariant derivatives of spherical geometry have been deduced using Mathematica with Weisstein (s.d.) as a guideline. A comprehensive presentation can be found in Malvern (1969, App. 1).

Another derivate useful to consider is local rotation (a.k.a. curl). A spheroidal-modes model of GIA like the one used in Milne et al. (2004) is not capable of producing curl; however a thin or a thick plate is. Thus, significant amounts of curl in the horizontal motion could indicate non-GIA conditions or an impact due to deviations of a radially nonsymmetric earth structure.

\subsection{Energy}

In the thick-plate adjustment observation error is traded against elastic and potential energy. The rate of energy $E$ that gets stored in a volume $d V$ is computed from the relation

$$
\frac{d E}{d t} d V=(\mathbf{f} \cdot \dot{\mathbf{u}}+\boldsymbol{\sigma}: \dot{\boldsymbol{\epsilon}}) d V
$$

where $\mathbf{f}$ is volume force density, $\dot{\mathbf{u}}$ displacement rate, $\boldsymbol{\sigma}: \dot{\boldsymbol{\epsilon}}$ the dyadic product of the tensors of stress and strain rate, respectively. In the case of work against the potential the force density $\mathbf{f} \propto-\mathbf{u} \hat{\mathrm{r}} \hat{\mathrm{r}}$. In a model with layered density, the contributions come from the density jumps $\delta \rho$ so that the force density at time $t$ may be approximated by

$$
f_{r}=-g \delta \rho\left(u_{r}+\dot{u}_{r} t\right)
$$

where $g$ is the ambient gravity acceleration. In a pure situation of a GIA process we could assume

$$
\dot{\mathbf{u}} \propto-\mathbf{u}
$$


However, such a relation holds srictly only for a single-mode approximation of the process. An exact, local relation between $\dot{\mathbf{u}}$ and $\mathbf{u}$ would require the introduction of a spatial-temporal filter in order to account for the dispersion of visco-elastic Love-numbers (the spectrum of relaxation time versus harmonic degree). However, this would still imply the assumption that all modes were excited by glacial loading. In trying to keep the thick-plate model as independent of GIA-assumptions as possible we rather assume a constant local proportionality. Therefore our buoyancy energy term will only take the product

$$
g \frac{t^{2}}{2} \int \delta \rho \dot{\mathbf{u}} \cdot \dot{\mathbf{u}} d V
$$

into account, surmising that we minimise the work of a plate as it departs from an undeformed state to the incremental deformation occurring in one unit of time.

Similarly, in the case of elastic energy we ignore the background stress and minimise only the work due to the stress increment of the ongoing deformation. Since the problem involves only a purely elastic plate, the principle of correspondence applies trivially.

\subsection{Model details}

Let $R$ denote the radius of the earth and take a quadrangle of width $\Delta \Lambda$ in longitude and $\Delta \Phi$ in latitude, centred at $\Lambda_{0}$ and $\Phi_{0}$, encompassing the area of interest. We formulate the 3D-displacements as a finite sum of Chebyshev polynomials

$$
\mathbf{u}(\xi, \eta)=\sum_{i=0, j=0}^{N, M} \mathbf{U}_{i j} T_{i}(\xi) T_{j}(\eta)
$$

with $\mathbf{u}=[u, v, w]$ vertical, south, east displacement and associated coefficients $\mathbf{U}_{i j}=\left[U_{i j}^{(k)}\right]$, $k=1,2,3$, at the surface of a thick elastic slab, $-1 \leq \xi \leq 1,-1 \leq \eta \leq 1$, longitude $\lambda=\Lambda_{0}+$ $\xi \Delta \Lambda / 2$, and colatitude $\phi=\Phi_{0}+\eta \Delta \Phi / 2$. When we consider a thick plate the displacements in (1) represent the neutral layer where the strain induced by bending vanishes, and the displacement along the surface, i.e. the result of bending, must be added. Here, we use Turcotte and Schubert (2002)

The thin-plate strain components and rotation $\omega_{z}$ are readily computed from Weisstein (s.d.)

$$
\begin{gathered}
\epsilon_{\phi \phi}=\frac{1}{R}\left(\frac{\partial v}{\partial \phi}+u\right) \\
\epsilon_{\lambda \lambda}=\frac{1}{R \sin \phi}\left(\frac{\partial w}{\partial \lambda}+\sin (\phi) u+\cos (\phi) v\right) \\
\epsilon_{\phi \lambda}=\frac{1}{2 R \sin \phi}\left(\frac{\partial v}{\partial \lambda}+\sin \phi \frac{\partial w}{\partial \phi}-2 \cos (\phi) w\right) \\
\omega_{z}=\frac{1}{2 R}\left(\frac{\partial w}{\partial \phi}-\frac{1}{\sin \phi} \frac{\partial v}{\partial \lambda}+\cot (\phi) w\right)
\end{gathered}
$$

and

$$
\begin{aligned}
\frac{\partial}{\partial \phi} & =\frac{2}{\Delta \Phi} \frac{\partial}{\partial \eta} \\
\frac{\partial}{\partial \lambda} & =\frac{2}{\Delta \Lambda} \frac{\partial}{\partial \xi}
\end{aligned}
$$


The leading terms of thick-plate strain at depth $z$ reckoned positive downward from a neutral layer $z=0$ are dependent on the vertical displacement

$$
\begin{gathered}
\epsilon_{\phi \phi}^{(\mathrm{TP})}=-\frac{z}{R^{2}}\left(\frac{\partial^{2} u}{\partial \phi^{2}}-\nu \frac{1}{\sin ^{2} \phi} \frac{\partial^{2} u}{\partial \lambda^{2}}\right) \\
\epsilon_{\lambda \lambda}^{(\mathrm{TP})}=-\frac{z}{R^{2}}\left(\frac{1}{\sin ^{2} \phi} \frac{\partial^{2} u}{\partial \lambda^{2}}-\nu \frac{\partial^{2} u}{\partial \phi^{2}}\right) \\
\epsilon_{\phi \lambda}^{(\mathrm{TP})}=-\frac{z}{R^{2} \sin \phi} \frac{\partial^{2} u}{\partial \phi \partial \lambda}
\end{gathered}
$$

with the Poisson ratio $\nu$. The Chebyshev expansion of the surface displacements and with it the strain is supposed to include the thick-plate action. Thus, the strain at depth $z$ becomes

$$
\epsilon(z)=\epsilon^{(\mathrm{S})}-\epsilon^{(\mathrm{TP})}(z)
$$

for any horizontal component, superscript S denoting the surface strain (Eq. 2-5).

The penalty function is formulated as follows

$$
\begin{aligned}
P & =\frac{1}{N_{\mathrm{obs}}} \sum_{i=1}^{N_{\mathrm{obs}}}\left(\tilde{\mathbf{u}}_{i}-\mathbf{u}_{i}\right)^{\top} \cdot \boldsymbol{\Sigma}^{-2} \cdot\left(\tilde{\mathbf{u}}_{i}-\mathbf{u}_{i}\right) \\
& +\frac{\Omega}{N_{e}} \sum_{i=1}^{N_{e}}\left[E\left(\phi_{i}, \lambda_{i}\right)+B\left(\phi_{i}, \lambda_{i}\right)\right]
\end{aligned}
$$

where the first term computes the normalised $\chi^{2}$ of the fit of observations $\tilde{\mathbf{u}}_{i}$ weighted by their variance-covariance matrix $\boldsymbol{\Sigma}$, the second term the elastic and the third the buoyancy energy. Each observation error is scaled with its standard deviation. The energy terms are scaled with $\Omega$, the energy penalty factor. $E$ consists of the in-plane and bending strain energy density (in N/m)

$$
E=\frac{1}{2} \int_{-T_{1} / 2}^{T_{1} / 2}\left[\boldsymbol{\sigma}: \boldsymbol{\epsilon}+\boldsymbol{\sigma}^{(\mathrm{TP})}(z): \boldsymbol{\epsilon}^{(\mathrm{TP})}(z)\right] d z
$$

where the colon signifies the dyadic product, and buoyancy energy density

$$
B=\frac{1}{2} g \rho_{\mathrm{eff}} u^{2}
$$

sampled at $N_{e}$ random points. The stress tensor $\boldsymbol{\sigma}$ is obtained from the strain tensor $\boldsymbol{\epsilon}$ by means of the Hooke-Lamé equations. As can be seen from equations (12) and (13), the energy terms allow lateral heterogeneity in elastic and density parameters. The orthogonality of the Chebychev polynomials under horizontal integration is not employed.

Finally, we search for a minimum of $P$ by varying the Chebyshev coefficients in the matrix U. Since all equations are linear in the coefficients, the partial derivatives of $P$ can easily be computed from the variation of $P$ when each coefficient is switched between zero and unity.

The uncertainties for the estimated quantities are computed by exploring the permitted observation misfit, the first term in (11), as follows. Varying each Chebyshev-coefficient we obtain a normalised $\chi^{2}$ that is larger than the best-fit value. We invert the equation to determine the 
value of the examined parameter such that the observation square-error is enlarged by a factor of 2 , spreading the individual contributions equally. While stepping through a regular longitudelatitude grid, the RMS of the deviations in each target quantity is summed up. Thus we obtain the RMS of all deviations (e.g. of areal strain rate) that in its entirety leads to a half-as-good observation fit.

Here's the method. Let $\mathbf{1}_{i j k}$ be a zero matrix except for a value of unity at $i j k$. Let further $q(\phi, \lambda, \mathbf{U})$ be a target quantity (e.g. areal strain) with $\mathbf{U}^{*}$ the $M \times N \times 3$ matrix of Chebyshevcoefficients pertaining to the best fit. Denote the observation penalty by $\chi^{2}$. Assume that varying coefficient $i j k$ by $\delta U_{i j}^{(k)}$ creates an increase of $\chi^{2}$ by $\delta \chi^{2}$.

$$
\chi^{2}\left(\mathbf{U}^{*}+\mathbf{1}_{i j k} \delta U_{i j}^{ \pm(k)}\right)-\chi^{2}\left(\mathbf{U}^{*}\right)=\delta \chi^{2}
$$

To obtain a value for $\delta U_{i j}^{ \pm(k)}$ we demand that

$$
\delta \chi^{2}=\alpha \frac{\chi^{2}\left(\mathbf{U}^{*}\right)}{3 M N}
$$

$\alpha=1$ for a doubling of the misfit. Equation (14) is solved iteratively with a root finder. Finally

$$
\sigma=\sqrt{\frac{1}{2} \sum_{i j k \pm}\left[q\left(\phi, \lambda, \mathbf{U}^{*}+\mathbf{1}_{i j k} \delta U_{i j}^{ \pm(k)}\right)-q\left(\phi, \lambda, \mathbf{U}^{*}\right)\right]^{2}}
$$

is our estimate of the uncertainty of $q$ at $\phi, \lambda$.

\section{Data}

The study will be carried out on two kinds of input data. First we use GIA model predictions (Milne et al., 2004) in order to supply a well known signal. At this stage we can analyse how well the discrete GNSS station network can retrieve the continuous field. The particular model selected has a lithosphere thickness of $120 \mathrm{~km}$, an upper mantle viscosity of $0.5 \times 10^{21} \mathrm{Pas}$ and a lower mantle viscosity of $5 \times 10^{21}$ Pas. It adopts the ice load history of Lambeck et al. (1998) for Fennoscandia and ICE3g (Tushingham and Peltier, 1991) for the remaining part of the globe.

This model provided the best fit to the GPS solution of Lidberg et al. (2009). It should be noted that the vertical velocities produced by the GIA model are in general somewhat lower; however, the fit to the 3-D motions did prefer this model probably because the northward velocities in the north are generally overpredicted by the suite of models, maybe as a consequence of an ice load not extending sufficiently into the Barents area. Therefore, a generally lower velocity scale has advantages in the fit.

Since GIA produces an uplift dome with large vertical rates we will also study the importance of thick-plate flexure for the horizontal strain rates at the surface. In a second stage we adjust the thick-plate model to the difference between model and BIFROST GNSS site velocities.

As the observation data set we use the GPS solution of Lidberg et al. (2009). The thick-plate model is adjusted to the difference of the GIA model velocities minus those of the GPS solution.

\section{Results and discussion}

The following computations will be examined more closely: (1) The response of the thin- and thick-plate models by forcing it to fit the GIA model predictions at the GNSS stations along with 
Table 1: Results of observation fit. For each variant of the modelling we show the weighted RMS of signal and residual including all sites. The maximum residual WRMS over the three components at the most problematic site is shown in bold, and the component that is most critical follows. The next three rows show the largest error in each component. GIA model designates the predictions of Milne et el. (2004) that best fit the GPS analysis of Lidberg et al. (2009).

\begin{tabular}{|c|c|c|c|c|c|c|}
\hline \multirow[t]{2}{*}{ Input } & \multicolumn{2}{|c|}{ WRMS all sites } & \multirow{2}{*}{$\begin{array}{l}\text { Max. WRMS } \\
\text { /site }\end{array}$} & \multirow{2}{*}{$\begin{array}{c}\text { Max. err. } \\
{[\mathrm{mm} / \mathrm{yr}] \pm 1 \sigma}\end{array}$} & \multirow[t]{2}{*}{ Comp. } & \multirow[t]{2}{*}{ Site } \\
\hline & Signal & Residual & & & & \\
\hline \multicolumn{7}{|l|}{ Thick plate } \\
\hline \multirow[t]{4}{*}{ GPS } & 15.3 & 0.95 & 3.52 & $-0.66 \pm 0.12$ & $\mathrm{U}$ & SUUR \\
\hline & & & 1.15 & $1.34 \pm 0.46$ & $\mathrm{U}$ & BORK \\
\hline & & & 1.15 & $0.79 \pm 0.10$ & $\mathrm{~N}$ & SMID \\
\hline & & & 0.48 & $0.72 \pm 0.08$ & $\mathrm{E}$ & BORK \\
\hline \multirow[t]{4}{*}{ GIA-model } & 14.0 & 0.07 & 0.16 & $-0.14 \pm 0.49$ & $\mathrm{U}$ & KEVO \\
\hline & & & 0.16 & $-0.14 \pm 0.46$ & $\mathrm{U}$ & KEVO \\
\hline & & & 0.00 & $-0.02 \pm 0.10$ & $\mathrm{~N}$ & SMID \\
\hline & & & 0.00 & $-0.02 \pm 0.08$ & $\mathrm{E}$ & BORK \\
\hline \multirow{4}{*}{$\begin{array}{c}\text { GIA model } \\
\text { - GPS }\end{array}$} & 4.3 & 0.95 & 3.50 & $-0.65 \pm 0.12$ & $\mathrm{U}$ & SUUR \\
\hline & & & 0.91 & $1.32 \pm 0.46$ & $\mathrm{U}$ & BORK \\
\hline & & & 0.91 & $0.59 \pm 0.10$ & $\mathrm{~N}$ & SUUR \\
\hline & & & 3.50 & $-0.65 \pm 0.08$ & $\mathrm{E}$ & BORK \\
\hline \multicolumn{7}{|l|}{ Thin plate } \\
\hline \multirow[t]{4}{*}{ GPS } & 15.3 & 0.86 & 3.52 & $-0.66 \pm 0.12$ & $\mathrm{U}$ & SUUR \\
\hline & & & 0.52 & $-1.13 \pm 0.46$ & $\mathrm{U}$ & SMID \\
\hline & & & 0.94 & $0.68 \pm 0.10$ & $\mathrm{~N}$ & SUUR \\
\hline & & & 3.52 & $-0.66 \pm 0.08$ & $\mathrm{E}$ & BORK \\
\hline \multirow[t]{4}{*}{ GIA model } & 14.0 & 0.06 & 0.13 & $-0.06 \pm 0.34$ & $\mathrm{U}$ & SKE0 \\
\hline & & & 0.13 & $-0.06 \pm 0.46$ & $\mathrm{U}$ & SKE0 \\
\hline & & & 0.00 & $-0.02 \pm 0.10$ & $\mathrm{~N}$ & SMID \\
\hline & & & 0.00 & $-0.02 \pm 0.08$ & $\mathrm{E}$ & BORK \\
\hline \multirow{4}{*}{$\begin{array}{c}\text { GIA model } \\
\text { - GPS }\end{array}$} & 4.3 & 0.86 & 3.51 & $-0.65 \pm 0.12$ & $\mathrm{U}$ & SUUR \\
\hline & & & 0.49 & $0.95 \pm 0.46$ & $\mathrm{U}$ & VANO \\
\hline & & & 0.74 & $0.54 \pm 0.10$ & $\mathrm{~N}$ & SUUR \\
\hline & & & 3.51 & $-0.65 \pm 0.08$ & $\mathrm{E}$ & BORK \\
\hline
\end{tabular}

the uncertainty of the GNSS observations. (2) The difference between the GNSS observations and the GIA model predictions. Table 1 summarises the success of the adjustments along with the maximum residual errors. The table also shows one run of the model where the GPS solution is fitted before subtracting the GIA predictions. Generally, the weighted RMS if the fits to observed arrive below unity, the fits to the GIA model far below unity owing to its smooth, long-wavelenth dominated velocity field. As might be expected, the thick-plate solution leaves a larger residual than the thin-plate variant; however, with the observation error penalty chosen, the increase is only slight, 0.95 versus 0.86 , so that the thick-plate solution might still be charaterised as observation-dominated.

Graphics for the resulting uncertainties of inferred strain components cannot be shown here because of space limitations. In short, the areal and shear strain uncertainties are typically on the order of $1 \times 10^{-9} / \mathrm{yr}$ in the areal component, $2 \times 10^{-9} / \mathrm{yr}$ in shear, and $0.5 \mathrm{nrad} / \mathrm{yr}$ in curl. The 
uncertainties become five times larger towards the edges of the model. These particular numbers apply to the fit of the thick plate to the residual motion.

\subsection{Fit to the GIA model predictions}

Both the thin- and thick-plate models can be fit to the GIA predictions with a weighted residual RMS much less than unity. The thick-plate penalty due to flexure is seen to smoothen the response in the thick-plate solution, reproducing more closely the features of the GIA model as seen in Milne et al. (2004) (cf. Fig. 1).
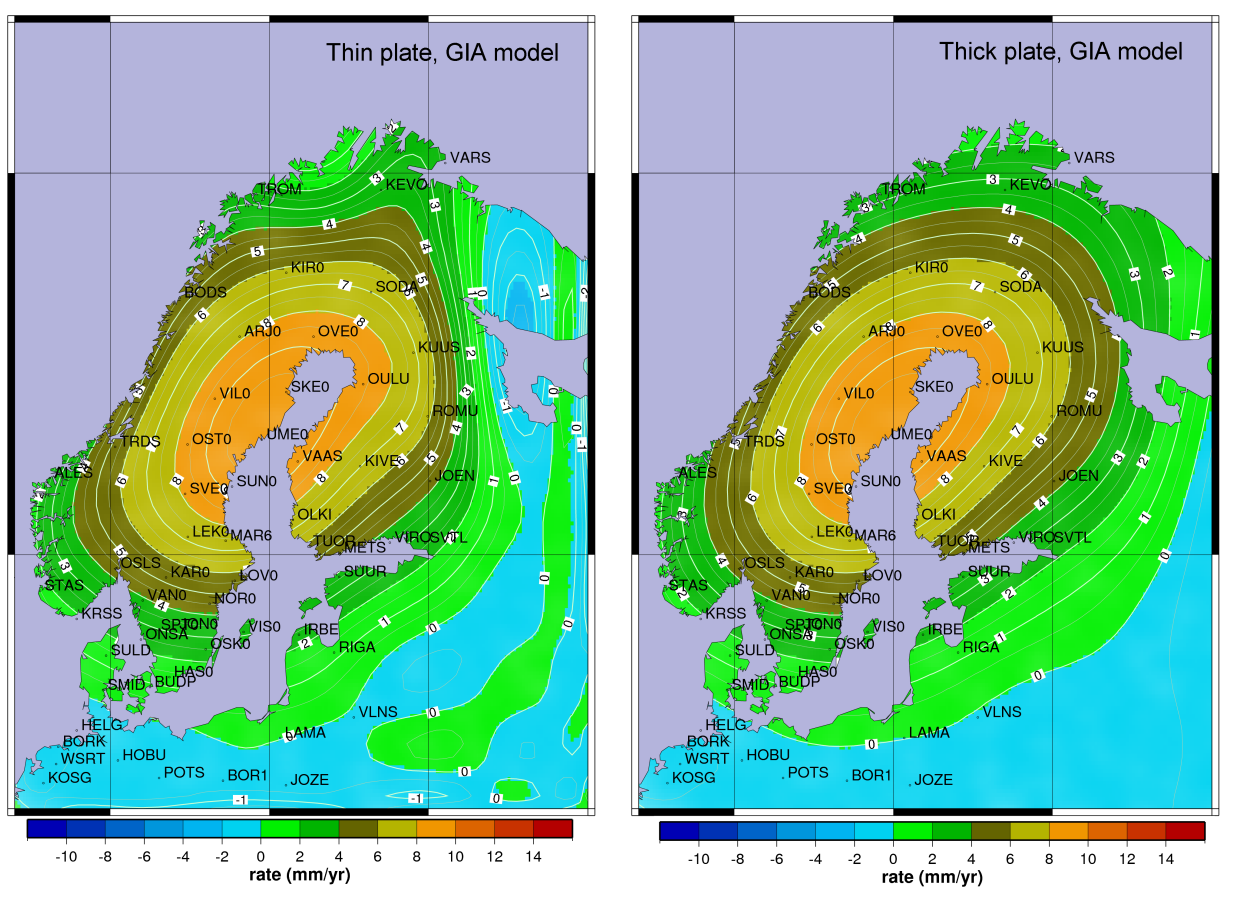

Figure 1: Vertical rates modelled with a thin plate (left) and a thick plate (right). The data fitted are the GIA predictions from Milne et al. (2004).

We show the deformation field in Fig. 2 where the thick-plate adjustment can be compared to the input GIA field.

The thin- and thick-plate solutions differ significantly as to the amount of in-plane strain at depth when vertical displacement is large as is the case when the model is fit to the GIA motion. Each extension due to flexure around one axis is accompanied by a compression at right-angle and scaled with the Poisson ratio. Thus the already smaller NE-SW extension is reduced further due to sharper bending around the NE-SW flexure axis. As a consequence, the mid-plane areal strain and the NW-SE strain component switch sign from extensional to compressional in the thick plate. Strain at the three depth levels is shown in Fig. 3.

Stepping beyong this simple model and contemplating the situation of viscous coupling of the asthenosphere when the isostatic rebound is in a phase of relaxation, the corresponding stress change would act in an opposite sense, extending the lithosphere rather than compressing. If the thick-plate model is correct the flexing lithosphere would increase the stress at the boundary 

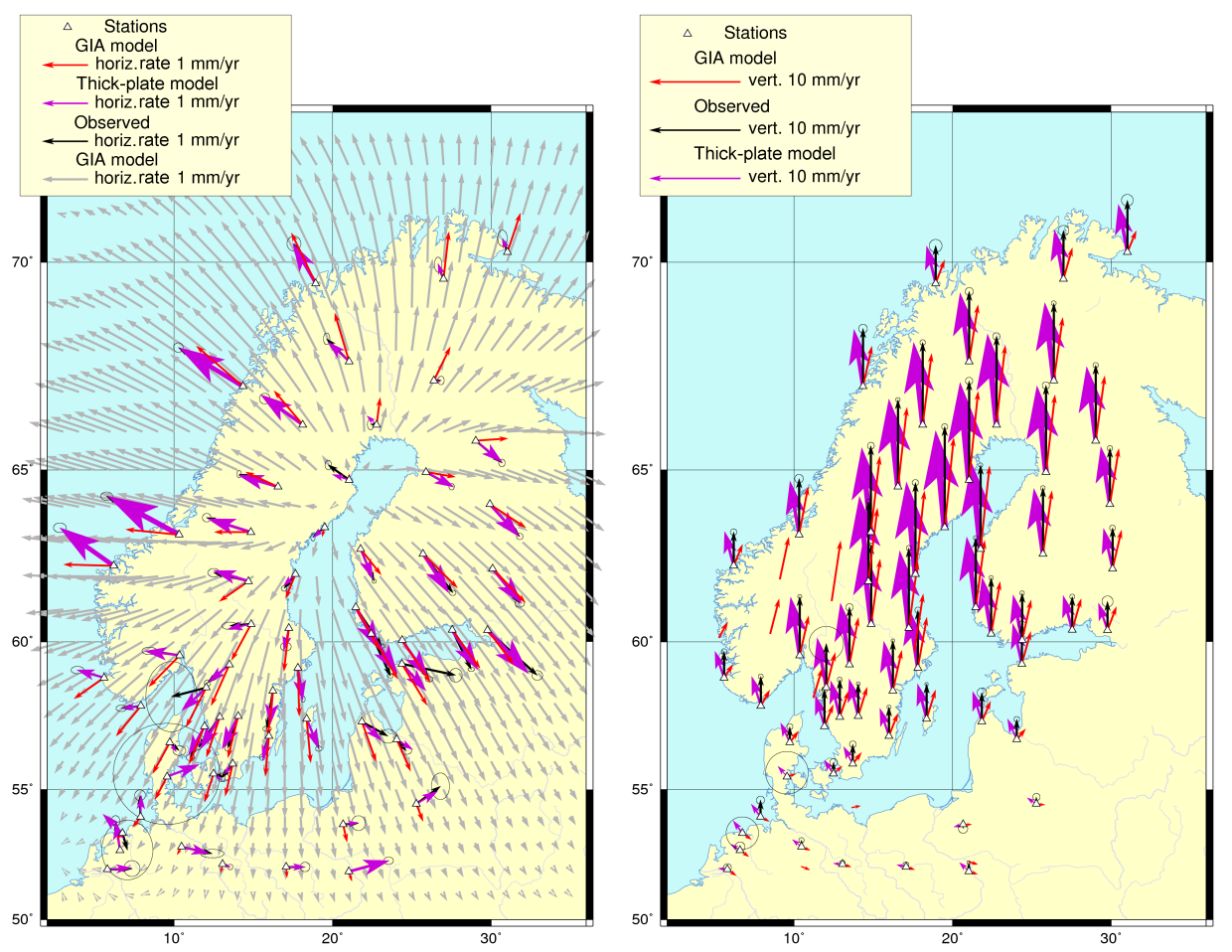

Figure 2: Displacement rates. Observed GPS solution from Lidberg et al. (2009), GIA predictions from Milne et al. (2004) and the fit to the GPS data using a thick elastic plate.

to the asthenosphere. This would lead to increased compression in the lower lithosphere and a downward shift of the neutral layer, and thus an increased extension at the surface in excess to what is computed in our thick-plate model that assumed horizontal stress-free conditions at the bottom of the slab. A more realistic vertical profile of horizontal stress in the lithosphere must be left to future development of the model.

The GIA predictions are based on a set of spheroidal base functions, and for that reason curl in the displacement field is zero. The "filtering" of the field through the GNSS network and its weighting produces a curl field that is in general less than the estimated uncertainty (cf. Fig. 3).

\subsection{Fit to the observed GIA model residual}

Two sites were reweighted owing to short records, assuming the long-term noise has not been averaged to the same level as in the longer records: SMID in Denmark and BORK on the North Sea island of Borkum, Germany. No further efforts have been taken to perform robustness tests or reweighting of some of the observations in the fit as the majority of WRMS contributions is near unity or below.

Examining the results of the plate models when they are fitted to the residual observations (GNSS minus GIA-model, see Fig. 4) we emphasize the following features. In the northern part of the area the GIA model overpredicts the observed horizontal motion. Southward motion at all stations north of latitude $67^{\circ}$ appears well correlated, excepting the two Norwegian sites BODS and TROM. To some extent this is a consequence of the sparseness of the GNSS network. With 


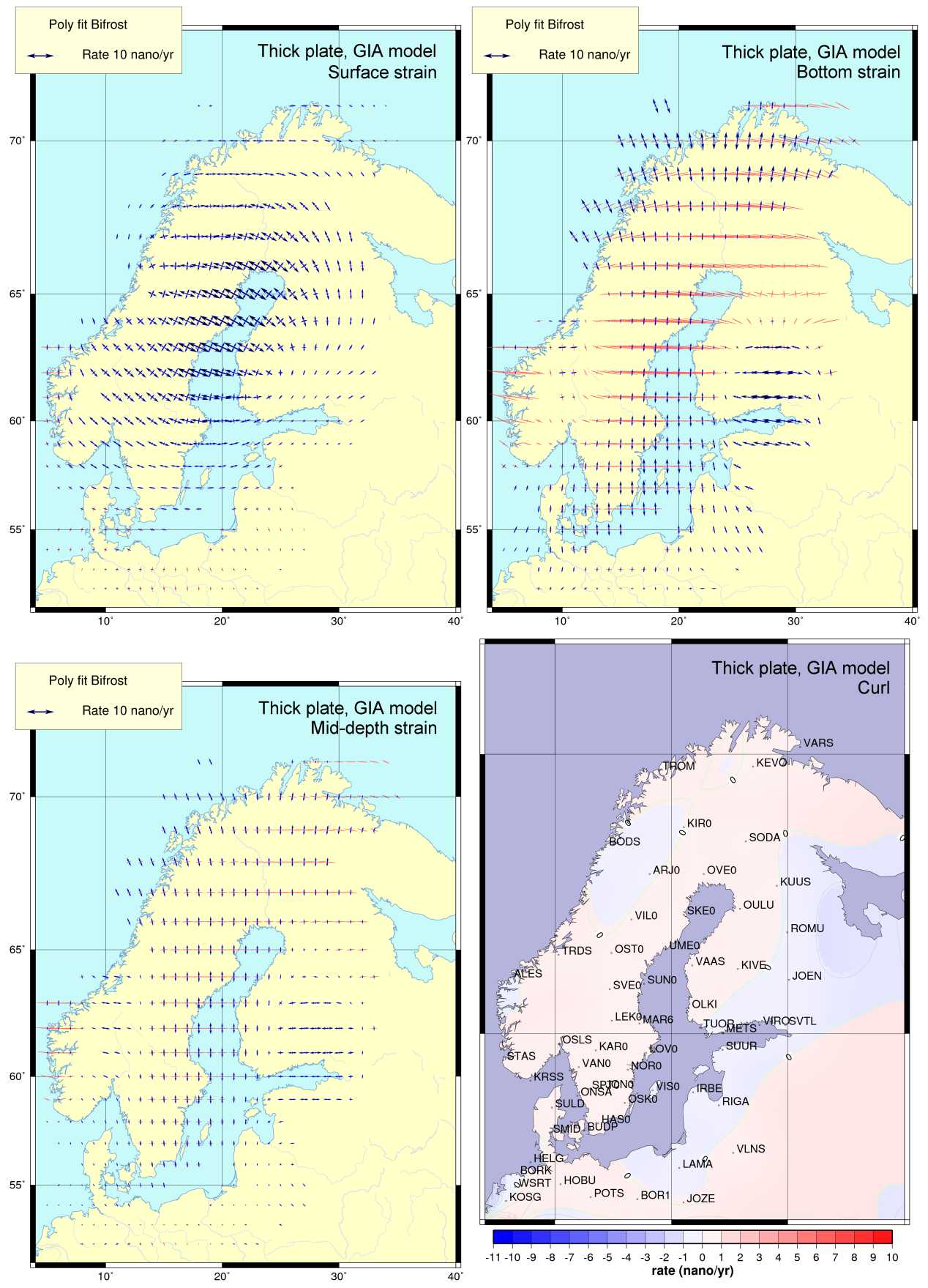

Figure 3: Strain rates computed by the thick-plate model at surface and bottom of the slab (top row) and at mid-plane depth (bottom left). Curl is show in the bottom right map. 

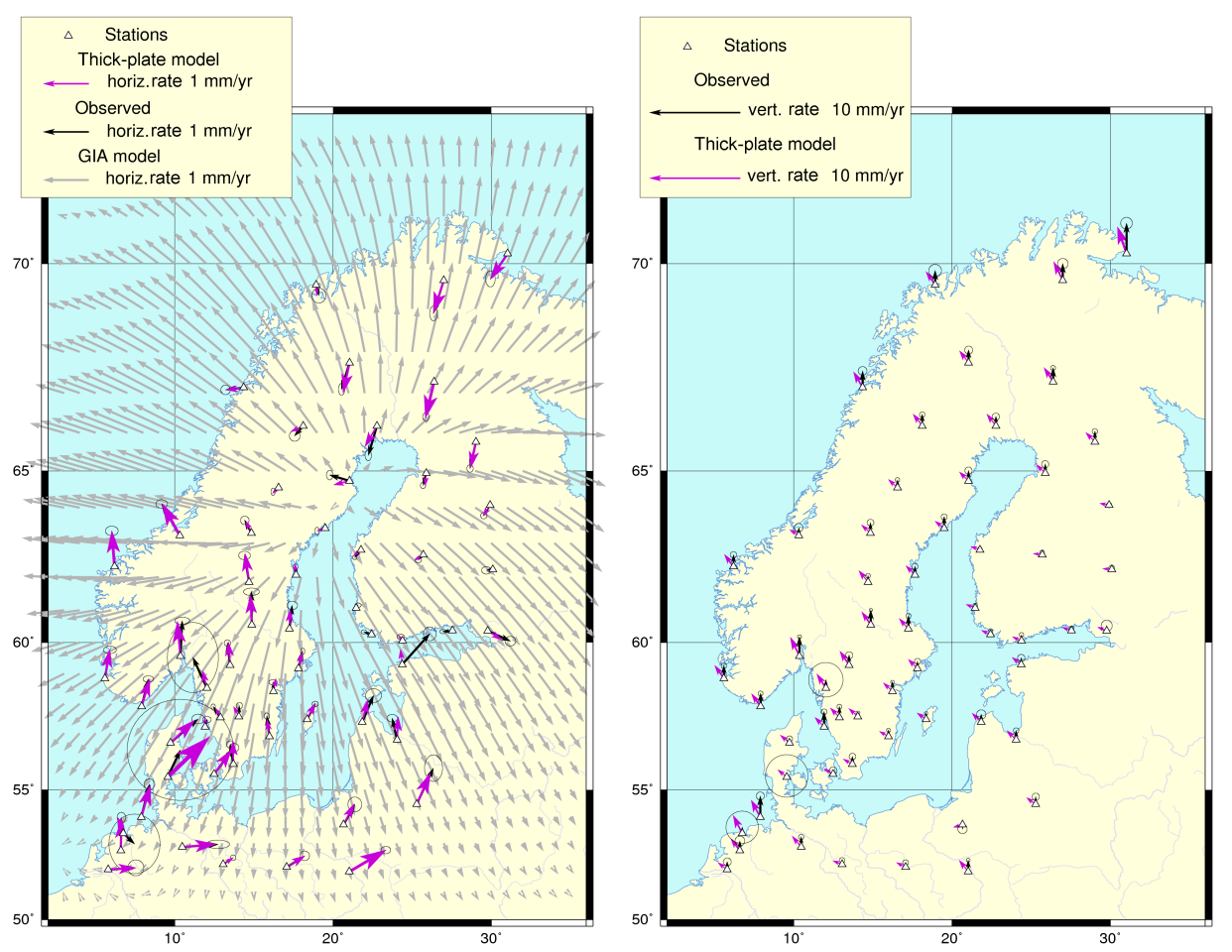

Figure 4: Residual motion (GPS minus GIA model), horizontal velocities left, vertical right, and the thick-plate solution.

more stations in the north the minimisation of the misfit would have distributed the residual more evenly over the region, since there are three degrees of freedom in the GNSS results with respect to the origin of global rotation. However, since the plate model is formulated in a spherical geometry, and since it has free-slip boundaries, we can rely on the absorption of a rigid rotation in the curl of the displacement field. One reason for the overprediction of northward rates in the North might be found in the extent of the ice load. With more ice load distributed towards the northern limit of the peninsula, the increase of horizontal rates would also be shifted towards more northern latitudes.

Figure 5 shows the thick-plate solution for surface strain and curl. Strain is also shown in the areal and shear components. The negative areal strain rates found throughout the central and northern parts of Fennoscandia suggests that the GIA model overpredicts the GPS results primarily in the aspect of areal strain. Typical rates are 4 nano/yr in areal and 6 nano/yr in shear, extension and compression both prevailing in north-south azimuth.

One conspicuous feature is found north of Kiruna, where areal strain rates reach levels of 10 nano/yr. It appears that the station rate of Tromso is relatively large towards the north. The thick plate responds by a pair of curl centres with clockwise rotation towards the east and counterclockwise towards the west while compression turns over to extension in between the curl dipole. Also shear strain obtains a local maximum, round 12 nano/yr, there. The area is one of the least well-resolved regions in the GNSS network. The question that arises, whether the horizontal motion of Tromso is more than a local phenomenon, would require at least two additional ob- 
serving sites in Finnmarken. A similar wish is expressed regarding the south-western Norwegian highland and fjords area.

Another noteworthy feature in an area well covered by the GNSS network is found in southwest Finland and in southern Sweden, where GIA rates underpredict the GPS velocities, and consequently a region of extension is detected. The highest strain rates are found either along the edges of the GNSS station network or in the region covering Belgium, the Netherlands, Northern Germany, Denmark and Poland. With respect to the uneven quality of station monumentation, ground coupling, length of time series in the latter areas we hesitate to conclude strong indications of localised crustal deformation.

\section{Conclusions}

We have obtained first results of strain rate determination from a GPS velocity solution by adjusting an elastic, thick plate in a simultaneous minimisation of elastic and potential energy, and observation misfit. One conclusion that appears important and safe is that flexure is an important contributor to the strain rates and their style in the plate, particularly at depth. However, since observations at mid-lithosphere depths and beyond cannot be assimilated, and since the time scale of only the glacial isostatic adjustment but not of any competing process, is well known, boundary conditions for the lower lithosphere had to remain unconstrained, preventing an inversion of the model with respect to e.g. the thickness of the lithosphere. Instead, the major virtue of the thick-plate model has been seen in exposing anomalies in the geodetic station motion, using strain as an indicator while striving for a physically meaningful, deformation energy related, objective for the minimisation.

Analysing the part of the observed motion that remains unexplained using a best-fitting model for glacial isostatic adjustment (GIA) we find conspicuous features in the strain rate field in areas where the station network is sparse. In most of the rebound area, however, residual strain rates are found at magnutides of typically 4 nano/year and up to 8 nano/yr. In the central area, where glacial isostatic adjustment is prevailing and where station density is highest, two regions are identified where strain rates are either under- or overpredicted by the GIA model, underprediction in the central and northern uplift area und overprediction in southern Finland and in southern Sweden. Shear strain rates are at maximum 6 nano/yr. Southern Finland appears to show the lowest signatures of shear.

Curl is confined to a range of $\pm 2 \mathrm{nrad} / \mathrm{yr}$ except in northern Finland and Finnmarken. In this area, also a strain rate maximum is found at a level of 12 nano/yr. However, owing to the sparseness of the GNSS network in the north and west, the impact of anomalous motion at the few sites in the topographic slope in the fjord regions of the Scandian mountain belt is high; more definite conclusions as to a complex deformation pattern in northern Sweden and Finland would require more observing sites. The error analysis of the plate models suggest uncertainties in the central areal of $1 \mathrm{nano} / \mathrm{yr}$ for strain and $1 \mathrm{nrad} / \mathrm{yr}$ for curl.

Critical continuation of this study may focus on several questions. Since normal stress on a radially symmetric earth is inefficient to cause curl, The shear- and curl feature in LaplandFinnmarken may either be an effect of non-GIA origin or be related to lateral heterogeneity. The large-scale features predominant in the areal strain rate field may be indicative of systematic over- and underpredicition of horizontal motion in the GIA model, which could be a consequence of the adopted ice load history. Again, more GNSS stations in Finnmarken, Swedish Lapland, and Northern Finland would be desirable. 


\section{Acknowledgments}

Two anonymous reviewers have helped to improve this manuscript. Graphics were produced with the GMT mapping tools (Wessel and Smith, 1998).

\section{References}

Ahjos, T.,Uski, M., 1992. Earthquakes in northern Europe in 1375-1989, Tectonophysics, 207, 1-23.

Cai, J., Grafarend, E.W., 2007. Statistical analysis of geodetic deformation (strain rate) from the space geodetic measurements of BIFROST Project in Fennoscandia. J. Geodyn., 43, 214-238.

Haas, R., Scherneck, H.-G., Gueguen E., Nothnagel, A., Campbell, J., 2002. Large-scale strain-rates in Europe derived from observations in the European geodetic VLBI network. EGU Stephan Mueller Spec. Publ. Ser., 2, 1-14.

Lambeck, K., C. Smither, and P. Johnston, 1998. Sea-level change, glacial rebound and mantle viscosity for northern Europe, Geophys. J. Int., 134, 102-144.

Lidberg M., Johansson, J.M., Scherneck, H.-G., Davis, J.L., 2006. An improved and extended GPS-derived 3D velocity field of the glacial isostatic adjustment (GIA) in Fennoscandia. J. Geodesy, 81, 213-230.

Lidberg M., Johansson, J.M., Scherneck, H.-G., Davis, J.L., 2009. Unknown title. J. Geodynamics, this issue.

Malvern, L.E., 1969. Introduction to the Mechanics of a Continuous Medium, Prentice-Hall, Upper Saddle River, N.J., 713 pp.

Milne, G.A., Mitrovica, J.X., Scherneck, H.-G., Davis, J.L., Johansson, J. M., Koivula, H., Vermeer, M., 2004. Continuous GPS measurements of postglacial adjustment in Fennoscandia: 2. Modeling results. J. Geophys. Res., 109, doi: 10.1029/2003JB002619.

Nocquet, J.-M., Calais, E., Parsons, B., 2005. Geodetic constraints on glacial isostatic adjustment in Europe. Geophys. Res. Letters, 32, doi: 10.1029/2004GL022174.

Nyst, M., Thatcher, W., 2004. New constraints on the active tectonic deformation of the Aegean. J. Gephys. Res., 109, doi: 10.1029/2003JB002830.

Spakman, W., Nyst, M.C.J., 2002. Inversion of relative motion data for estimates of the velocity gradient field and fault slip. Earth Planet. Sc. Lett., 203, 577-591.

Turcotte, D.L., Schubert, G., 2002. Geodynamics, 2nd ed., Cambridge University Press, New York, 456pp.

Tushingham, A. M., and W. R. Peltier, 1991. ICE-3G: A new global model of late Pleistocene deglaciation based on geophysical predictions of postglacial relative sea level change. J. Geophys. Res., 96, 4497-4523.

Weisstein, E., (s.d.). http://mathworld.wolfram.com/ ChristoffelSymboloftheSecondKind.html

Wells, D.J., Coppersmith, K.J., 1994. New empirical relationships among magnitude, rupture length, rupture width, rupture area, and surface displacement. Bull. Seism. Soc. Am., 84, 974-1002.

Wessel, P. and W. H. F. Smith, 1998. New, improved version of the Generic Mapping Tools released, EOS Trans. AGU, 79, 579 . 


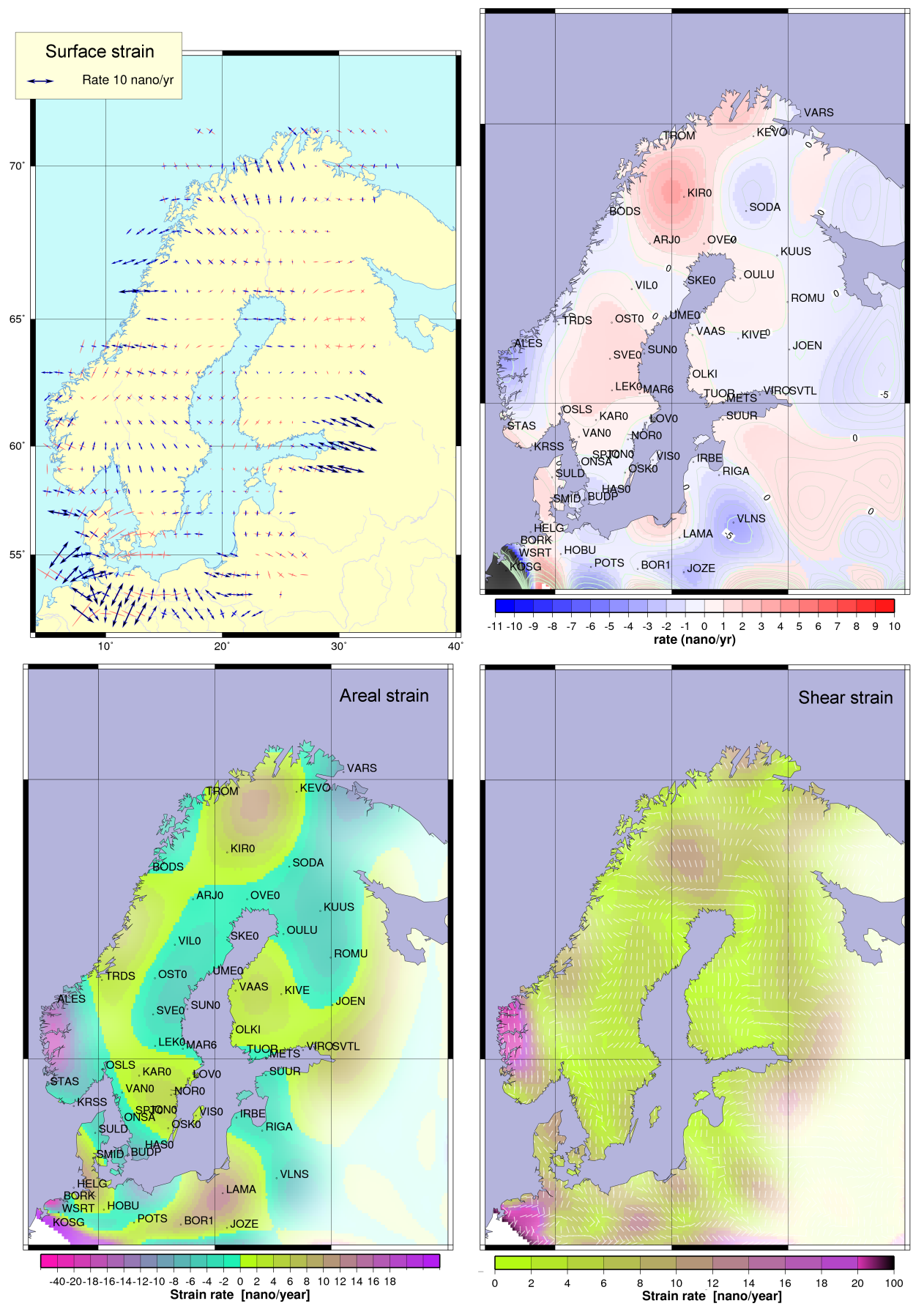

Figure 5: Thick-plate solution for surface strain rates (upper left) and curl (upper right), derived from the residual observed motion (i.e. after subtracting the GIA predictions. Strain decomposed into areal and shear is shown in the lower row. The maximum principal strain direction is shown as white stipples. 